\title{
Timber specimens parametrized design for numerical analysis
}

\author{
E. Martín Gutiérrez, J. Estévez Cimadevila, D. Otero Chans \& \\ S. Muñiz Gómez \\ Department of Technology of Construction, A Coruña University, Spain
}

\begin{abstract}
The characterization of materials or the study of resistant response associated with new constructive solutions often convey progressive adjustment of models of analysis starting from the results obtained by means of test campaigns. The reliability of the conclusions will obviously be conditioned by the amplitude of the experimental development which, as a result, should contain a sufficiently representative sampling of cases. In the situations where significantly multiple parameters intervene, specimens that involve possible values and combinations should be examined, which in practice remarkably increases the number of specimens to be treated. The case being presented deals with the evaluation of the effectiveness of certain fixed joints carried out by gluing metallic bars into timber pieces by means of adhesives of different formulations. The set of calculus patterns, including the extensive variability of the associated magnitudes, has been treated in a parametrized form. The geometry of the studied pieces, as well as the sustenance conditions and the load, are automatically generated by means of software specially designed for those purposes. The resulting information is structured in an orderly way to facilitate its reading and also its manipulation if needed. The files of the process obtained in this way can optionally incorporate the meshing sequences, as well as the commands of the calculus, analysis and postprocess. The adopted strategy considerably reduces the time assigned to the phases of the geometrical definition and resolution, and enormously simplifies the interpretation of the results.
\end{abstract}

Keywords: numerical analysis, parametrized design, glued joints. 


\section{Introduction}

This work is placed in an investigation project directed to the study of the joints behaviour in timber pieces by means of steel bars glued-in with an adhesive. The approach causes the development of two parallel complementary lines of advance: the first one is of experimental nature and the second one is based on the use of the numerical analysis technique.

On the one hand it is pretended to use the results obtained in the experiments for calibrating gradually the theoretical models in such a way that they, in their turn, could be applied for extrapolating not specifically experimented situations or facilitating the adoption of design decisions in certain practical cases. On the other hand the numerical analysis can be useful for the selection of those mechanisms of connection that prove to be more operative in advance, reducing the temporary and economic costs associated with the creation of the specimens.

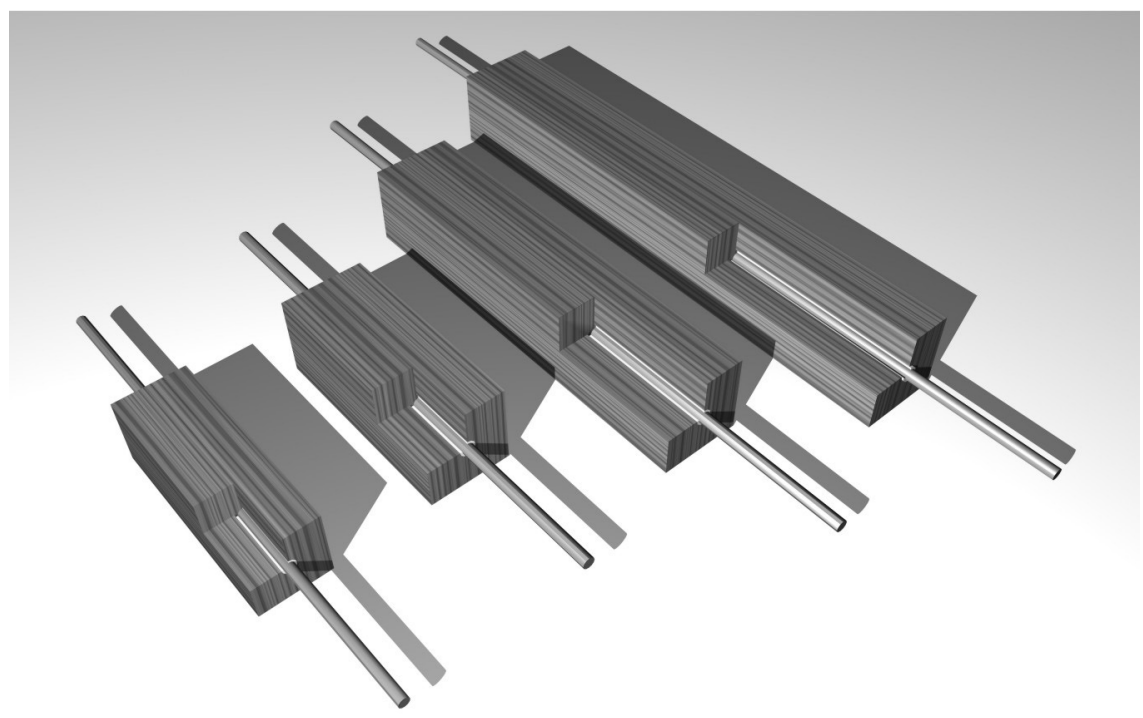

Figure 1: Reconstruction of different specimens.

Specimens are timber prisms of square transversal section made of timber, at both ends of which two threaded bars are placed, properly fixed by means of adhesives of epoxi formulation (fig. 1). The resulting specimen is subjected to a failure test of growing centered tensile load, registering the limit number and the form of the dominant failure. Throughout the process the equipment registers the total relative displacement between the ends of the specimen, associated to each load value, which later permits to configure the corresponding cause-effect diagrams. In principle a first experimental campaign consisting of a total of 45 pieces for each of the used adhesives is planned. The given number is distributed in 3 series of 15 specimens in which the joint with 3 possible diameters $(8,10$ and $12 \mathrm{~mm}$ ) and 5 anchorage lengths are analyzed (table 1). 
Table 1: The structure of the first experimental campaign.

\begin{tabular}{|c|c|c|c|c|c|c|c|c|c|}
\hline d & D & $\mathbf{e}$ & $\mathbf{L}$ & $\mathbf{L i}$ & $\mathbf{L m}$ & Le & Lb & $\mathbf{a}$ & Reference \\
\hline & & & & $L i=L$ & $3 . L \leq 500$ & & & $a=6 . d$ & Units: $\mathrm{mm}$ \\
\hline \multirow{5}{*}{8} & \multirow{5}{*}{10} & \multirow{5}{*}{1} & 60 & 60 & 180 & \multirow{5}{*}{150} & 210 & \multirow{5}{*}{48} & $\equiv 1 \mathrm{a} \varnothing$ \\
\hline & & & 90 & 90 & 270 & & 240 & & $\equiv 1 \mathrm{~b} \varnothing$ \\
\hline & & & 120 & 120 & 360 & & 270 & & $\equiv 1 \mathrm{c} \varnothing$ \\
\hline & & & 150 & 150 & 450 & & 300 & & $\equiv 1 \mathrm{~d} \varnothing$ \\
\hline & & & 180 & 140 & 500 & & 330 & & $\equiv 1 \mathrm{e} \varnothing$ \\
\hline \multirow{5}{*}{10} & \multirow{5}{*}{12} & \multirow{5}{*}{1} & 60 & 60 & 180 & \multirow{5}{*}{150} & 210 & \multirow{5}{*}{60} & $\equiv 2 \mathrm{a} \varnothing$ \\
\hline & & & 90 & 90 & 270 & & 240 & & $\equiv 2 \mathrm{~b} \varnothing$ \\
\hline & & & 120 & 120 & 360 & & 270 & & $\equiv 2 \mathrm{c} \varnothing$ \\
\hline & & & 150 & 150 & 450 & & 300 & & $\equiv 2 \mathrm{~d} \varnothing$ \\
\hline & & & 180 & 140 & 500 & & 330 & & $\equiv 2 \mathrm{e} \varnothing$ \\
\hline \multirow{5}{*}{12} & \multirow{5}{*}{14} & \multirow{5}{*}{1} & 60 & 60 & 180 & \multirow{5}{*}{150} & 210 & \multirow{5}{*}{72} & $\equiv 3 \mathrm{a} \varnothing$ \\
\hline & & & 90 & 90 & 270 & & 240 & & $\equiv 3 \mathrm{~b} \varnothing$ \\
\hline & & & 120 & 120 & 360 & & 270 & & $\equiv 3 \mathrm{c} \varnothing$ \\
\hline & & & 150 & 150 & 450 & & 300 & & $\equiv 3 \mathrm{~d} \varnothing$ \\
\hline & & & 180 & 140 & 500 & & 330 & & $\equiv 3 \mathrm{e} \varnothing$ \\
\hline
\end{tabular}

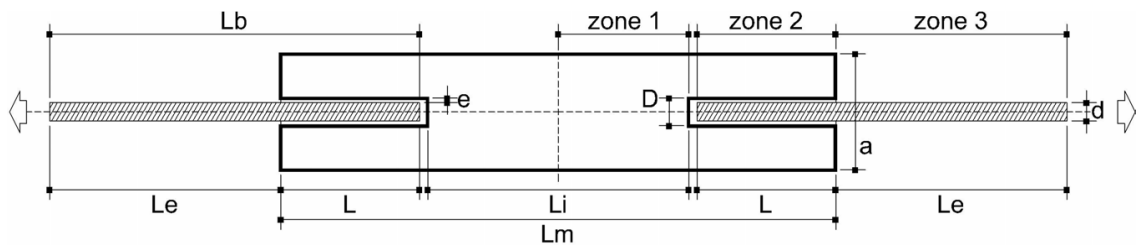

Figure 2: Initial configuration of the specimens.

The attached diagram shows the proposed combinations for the 9 geometrical parameters to deal with, as well as the type of the timber and the type of the applied adhesives. This set appears to be sufficiently full for establishing relevant conclusions in relation with the incidence of each magnitude and even facilitating formulations that will permit to define the necessary anchorage lengths.

\section{The structure of the process file}

The numerical models are calculated with the help of the application of Ansys Multiphysics, Swanson Analysis Systems Inc., initially assuming an elastic and lineal behaviour, noticeably correct in accordance with the experimentally obtained stress-deformation diagrams for load values not surpassing the 75-80 per cent of the limit number.

The volume of the pieces, the possible parametrization of its geometrical and mechanical values lead us to the development of a specific software that 
automates the definition of the model, taking advantage of the common commands of the preprocessor. It deals with the creation of a process file for each specimen that Ansys could manage in an autonomous way but in which the information would be structured in an orderly way with the purpose of simplifying its reading and the possible manipulation. In general the mentioned files respond to the following configuration: numerical definition of parametrized data; meshing specifications; assignment of the load value; access to the preprocessor of the system; description of the conditions of visualization of the model; definition of elements and materials; an orderly localization of the keypoints; positions that designate lines and arcs; creation of areas; construction of volumes by means of extrusion operations; material assignment; meshing operations; access to the calculus module; definition of coercion and application of the load; access to the postprocessor of the application; energy estimation of errors structured by materials; and presentation of results.

It should be paid attention to the fact that the file in question is defined in ASCII format, therefore it turns out to be operable with any conventional line editor. This circumstance allows to assess, with remarkable simplicity, diverse alternatives of modelling, for example: typology of the involved finite elements; adequacy of the parameters that define the mechanical behaviour of the materials; variations in the conditions of the meshing and adjustment of dicretization in concrete areas.

The model is configured by means of tridimensional elements of 8 nodes (Solid45), that present 3 degrees of liberty in each position (translations $\mathrm{x}, \mathrm{y}, \mathrm{z}$ ). After diverse experiments the use of elements of 20 nodes has been discarded, taking into consideration the fact that it greatly increases the storage requirements and the time of the calculus without significant differences neither in the results nor in the reductions in the energy estimations of the possible committed errors. The associated materials are: steel $8.8\left(\mathrm{f}_{\mathbf{y}}=640 \mathrm{~N} / \mathrm{mm}^{2}, \mathrm{f}_{\mathrm{u}}=800\right.$ $\mathrm{N} / \mathrm{mm}^{2}$ ) for the threaded bars of the ends, sawn timber (basically chestnut) and 3 types of adhesives of epoxi formulation (Sika AnchorFix-3, Hilti Hit Re-500, Loctite Hysol 9464A\&B). All of them are integrated in the model by their parameters of elastic behaviour; basically the modules of transversal and longitudinal elasticity and the coefficient of Poisson in relation with the axes that define the Cartesian system of reference (is designated as $Z$ the relative to the direction of the load application). The mentioned magnitudes are initially decided on the basis of the recommended values of different references $[1,2,6]$, and considering the experiments previously made upon the isolated materials. Later a successive adjustment is realized with a purpose of approximating the obtained results by means of experimental and numerical technique.

\section{Geometrical organization of the model}

Attending to the considerations of symmetry it is decided to define a fraction corresponding to the eighth part of the specimen. At first, the configuration of the generic transversal section is established that will give place to the complete volume of the modelling by repetition of its elements, totally or partially. In 
principle, ample divisions are managed to tackle the first assessment of the problem. Later comes the restructuring of the configuration on the basis of a radial scheme that serves to a better estimation of the stress distribution in different materials, especially in the areas close to the contact surfaces (fig. 3).

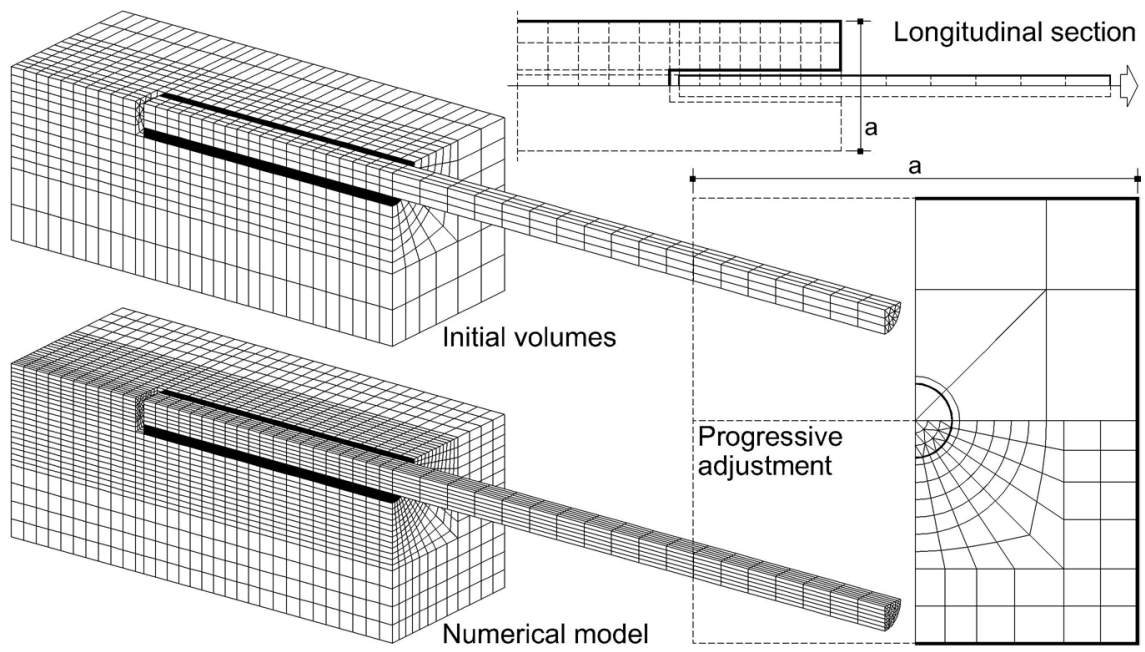

Figure 3: $\quad$ Sections and axonometry of the model.
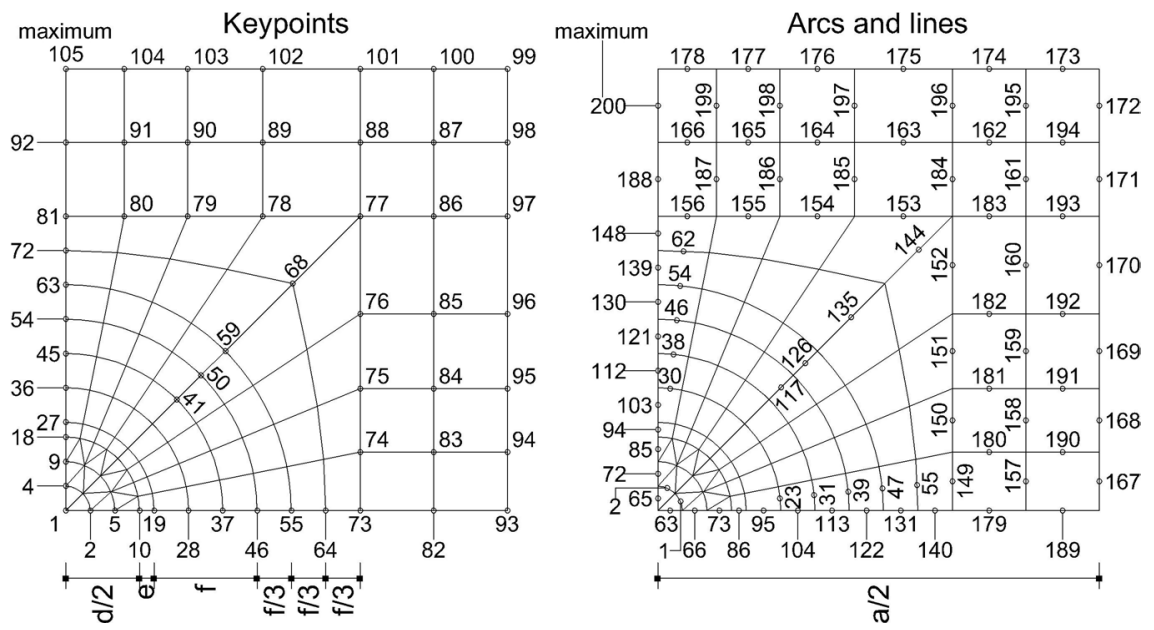

Figure 4: Basic numeration of nodes, lines and arcs.

At the beginning of the process the coordinates (x,y) of the 105 points, that define the base plane, are stored in matrices. In the area nearest to the centre of the section the mentioned values are deduced by means of a polar system of reference, while in the exterior panels a Cartesian type of analysis is proposed. It should be paid attention to the fact that the parametrization of the process of 
discretization permits the successive application of the adjustments that will improve the behaviour of the model.

The generation program supposes that the specimen is formed by 3 well differentiated areas (fig. 2): the central zone (zl), corresponding integrally to the timber piece; the relative to the glueline (z2); and the external fraction of the steel bar (z3). Each of these sets can be subdivided independently in the desired number of bands. With these premises the definition of the model keypoints is realized by means of the command:

\section{K,NPT (reference number for keypoint),X,Y,Z (keypoint location)}

The generation sequences of the nodes are structured by means of nested buckles for each of the described areas: an external one that runs across all the fringes of the zone that has been fractioned; and another internal one that analyzes the 105 constituting points of each transversal section (18 in the third sector). In each case the number of the node is resolved by means of a counter, the coordinates $(\mathrm{x}, \mathrm{y})$ are deduced from the previously formed auxiliary matrices, and the position of $(\mathrm{z})$ is estimated in the external buckle in relation with the section that is being configured at every moment. The following phase supposes the creation of lines and arcs with an arrangement corresponding respectively to the orders:

\section{L,P1,P2 (keypoints at the end of the line or circular arc line) LARC,P1,P2,PC (center), RAD (radius of curvature of the arc)}

In this case, Ansys does not require the numeration of such entities in the command line, that is why it is considered as appropriate to specify it as a comment to its term. This possibility simplifies, in its case, the localization of the elements in the list. The process is configured equally by means of buckles, updating in each transversal plane the number of its lines and arcs with a total arrangement of the previously treated sections.

Once the bases of the wire structure of the model are formed, the definition of the areas based on the lines and the arcs that configure its perimeter is realized. The process is very similar to the described one in accordance with the previous phase and is used in any case of the command:

A,P1,P2,P3,P4,P5,P6,P7,P8,P9 (list of keypoints defining area)

For configuring the volumes an extrusion of the definite areas in each section is realized in such a way that the numeration of both types of entities turns out to be coincident (fig. 5). To achieve this operation it is necessary to create in advance auxiliary lines over the axis of the specimen, linking the central nodes (1) of each of the consecutive two sections. The order that formalizes the extrusion responds to the following syntax:

VDRAG,N1,N2,N3,..,,N6 (areas in the pattern),NLP (line defining the path) 


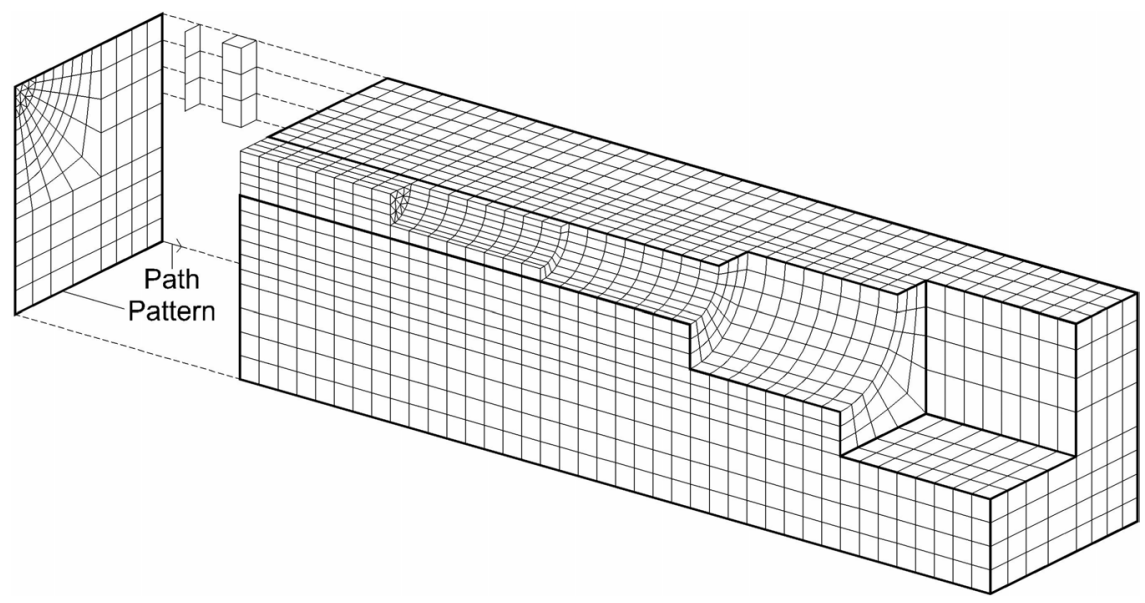

Figure 5: Obtaining volumen by extrusion.

At the end of this phase the volumes that are situated between the zones $\mathrm{z} 1$ and $\mathrm{z} 2$ (additional band of $2 \mathrm{~mm}$ ) relative to the bottom of the bar and the adhesive are eliminated. This elimination is decided after the real specimens are analyzed with a very reduced efficiency of the adhesive in this area, as a consequence of the filling process itself. In figure 4 the resulting slot caused by the elimination commands is estimated (VDELE, NV1, NV2, NINC: delete volumes from NV1 to NV2 in steps of NINC).

To finish the preprocess of the model, the obtained volumes are related to the involved materials, a task in which controlling the numeration of the first items proves to be fundamental. The selection is realized by means of ranks, a reason for which in each section at first the central steel elements are coded, then the relatives to the band of the adhesive and finally the constituent elements of the timber.

VSEL,Type,Item,,VMIN,VMAX (values of the item range),VINC (increment) VATT,MAT,REAL,TYPE (parameters to be assigned)

The meshing only affects the final volumes (VMESH,ALL). With a previous discretization, a subdivision of each edge in two halves (ESIZE,2) is considered correct. The application permits to refine the mesh in determined areas, but advanced studies indicate that the resultant model is sufficiently approximate without further modifications of the set.

\section{Analysis and interpretation of the results}

In the module of the calculus the transversal displacements coerce the symmetry planes (used for reducing the real specimen to its eighth part). So the external load is applied to the extreme surface of the steel bar, and the phase of analysis 
begins. After the calculus is finished, the postprocessor is opened studying the magnitudes that define the solution: basically the energy estimation of the errors, displacements (fundamental for its comparison with the experimental results), and stress conditions (fig.6). An important question is the distribution of the tangential stresses over the surface of contact between the adhesive and the timber, a parameter that could be determinant in numerous extreme situations (fig.7). For that reason the nodes of the mentioned area included in the plane (xz) are isolated and a list of resisting normal and tangential stresses is requested. A third computer program extracts the desired values ( $\tau_{x z}$ in this case) and starts to represent them in an environment of computer aided design (fig. 8).

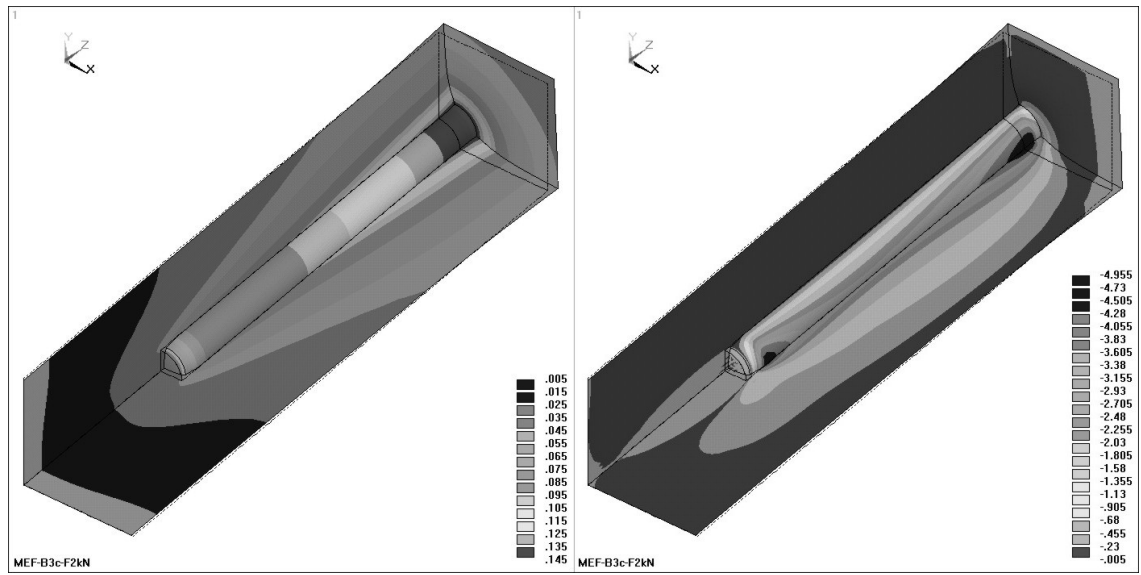

Figure 6: Displacements $(\mathrm{z})$ and tangencial stresses $\left(\tau_{\mathrm{xz}}\right)$.

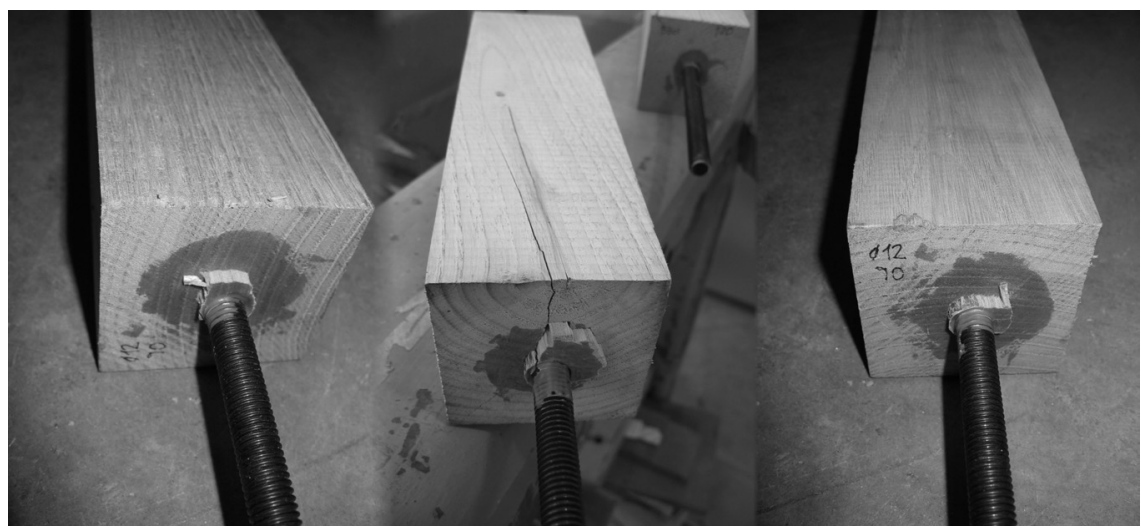

Figure 7: $\quad$ Failures associated with the excessive tangential stresses.

The diagrams show curved lines with maximal numbers focused on the ends of the anchorage area which are much greater than the average value traditionally used in the references. Both peaks turn to be similar for reduced longitudes, 
increasing noticeably the exterior one when the union is realized with more profundity.
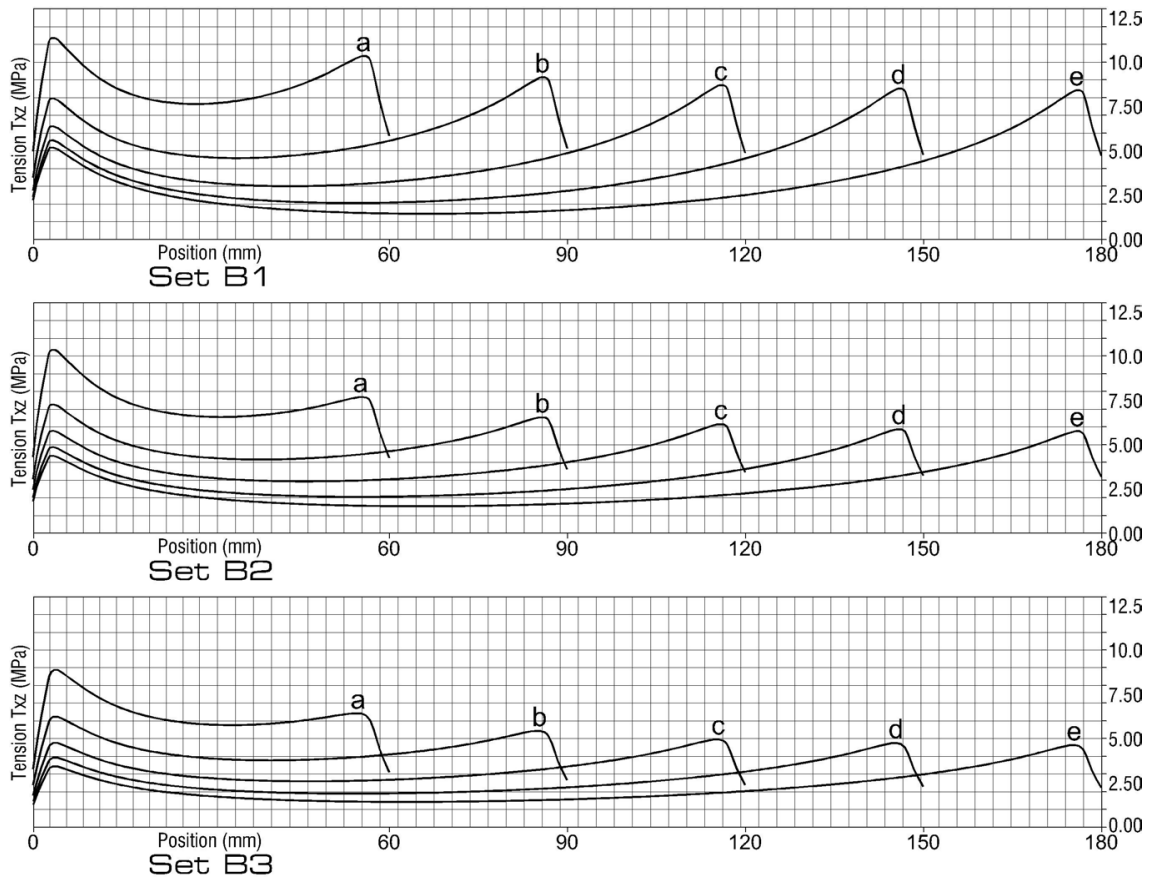

Figure 8: Distributions of the tangential stress.

\section{Conclusions}

The means in parallel to experimental technique and numerical analysis, for the study of the resistant behaviour of timber-steel joints as the described ones, supposes a sufficiently complete and illustrative strategy. The comparison of the results allows to calibrate gradually the virtual models in such away that they prove to be useful for verifying practical cases that are not specifically subjected to tests, or for predicting possible situations of risk. Likewise, the mentioned idealizations are useful for assessing constructive solutions at the very beginning, thus reducing the relative costs of production and of the load of possible prototypes. In this generic frame the computerized configuration of the models remarkably simplifies the generation and postprocess tasks appealing to the parametrization of its geometry.

\section{Acknowledgements}

This research is sponsored by the Ministry of Science and Technology through research project title "uniones metálicas encoladas con adhesivos en barras de 
madera" (glued anchoraged timer joints). The financial support is gratefully acknowledged.

\section{References}

[1] Broughton, J.G. \& Hutchinson, A.R. Effect on timber moisture content on bonded-in rods. Construction and Building Materials, volume 15, issue 1, pp. 17-25, 2001.

[2] Deng, J.X., Moss, P.J. \& Buchanan, A.H. Glued bolts in glulam. An analysis of stress distribution. $5^{\circ}$ World Conference on Timber Engineering, Montreux, Switzerland, 1998.

[3] Estévez, J., Pablos, J., Muñiz, S., Freire, M., Vázquez, R. \& Álvarez, J. Double-layer space structures of laminated timber tubular members. Proc. of the $4^{\circ}$ International Conference on Space Structures, University of Surrey, Guildford, UK, Ed. Thomas Telford Ltd.: London, pp. 563-572, 1993.

[4] Estévez, J. \& Vázquez, J.A. Spatial truss of hollow bars made of laminated timber supported by walls of reinforced masonry. Journal of the International Association for Shell and Spatial Structures, volume 45, n.1, April n.144.

[5] Estévez, J., Vázquez, J.A. \& Otero, M.D. Diseño y dimensionado del nudo extremo de una barra hueca de madera laminada. $1^{\circ}$ Congreso Ibérico A Madeira na Construçao, Universidade do Minho, Guimaraes, Portugal, pp. 689-698, 2004.

[6] Senno, M., Plazza, M. \& Tomasi, R. Axial glued-in steel timber jointsexperimental and numerical analysis. Holz als Roh - und Werkstoff, volume 62, number 2, 2004.

[7] Swanson Analysis Systems, Inc. Ansys User's Manual. Houston, 2005. (See also http://www.ansys.com). 